\title{
General mechanism for negative capacitance phenomena
}

\author{
J. Shulman, ${ }^{1,2, *}$ Y. Y. Xue, ${ }^{1,2}$ S. Tsui, ${ }^{1,2,3}$ F. Chen, ${ }^{1,2}$ and C. W. Chu ${ }^{1,2,4,5}$ \\ ${ }^{1}$ Department of Physics, University of Houston, 202 Houston Science Center, Houston, Texas 77204-5002, USA \\ ${ }^{2}$ Texas Center for Superconductivity, University of Houston, 202 Houston Science Center, Houston, Texas 77204-5002, USA \\ ${ }^{3}$ Department of Physics, California State University-San Marcos, 333 S. Twin Oaks Valley Road, San Marcos, California 92096, USA \\ ${ }^{4}$ Lawrence Berkeley National Laboratory, 1 Cyclotron Road, Berkeley, California 94720, USA \\ ${ }^{5}$ Hong Kong University of Science and Technology, Kowloon, Hong Kong
}

(Received 4 June 2009; revised manuscript received 25 August 2009; published 20 October 2009)

\begin{abstract}
The existence of a negative static dielectric constant has drawn a great deal of theoretical controversy. Experimentally, one has never been observed. However, low-frequency negative capacitance has been widely reported in fields including physics, chemistry, biology, geology, and electronics. This wide variety of systems possesses an extremely diverse set of physical processes that, surprisingly, share similar characteristics. We present a general mechanism that unites the various instances of negative capacitance under a common framework. The mechanism demonstrates that the negative capacitance arises from dc/ac signal mixing across a nonlinear conductor. Verification of the model is performed in physically distinct samples: an electrorheological fluid, a fuel cell, and a solar cell. Furthermore, we argue that the negative capacitance, under appropriate conditions, can be associated with a negative-differential dielectric constant, possibly even in the static limit.
\end{abstract}

DOI: 10.1103/PhysRevB.80.134202

PACS number(s): 73.63.-b, 72.20.Ht, 77.22.Gm, 82.70.Dd

\section{INTRODUCTION}

Materials and devices that exhibit negative capacitance (NC) are beginning to become quite common. It has been observed in samples as different as crystalline and amorphous semiconductor devices, ${ }^{1-9}$ organic compounds, ${ }^{10-14}$ composite materials/nanomaterials,,${ }^{9,15-22}$ electrochemical cells, ${ }^{23-25}$ geological samples, ${ }^{26}$ biological membranes, ${ }^{27-29}$ and even moist surfaces. ${ }^{30}$ Furthermore, it has been shown that NCs can originate at interfaces as well as in the bulk of the materials. However, they do typically share several common features: they arise in the presence of a dc bias; the $I-V$ characteristic is superlinear, i.e., the in-phase conductance $1 / R_{\mathrm{ac}}$ increases with the dc bias; and they possess a strong dispersion, such as a plasmalike admittance $1 / R_{\mathrm{ac}}+i \omega C \propto \frac{1}{1+i \omega \tau}$, where $\omega$ and $\tau$ are the frequency and a time scale, respectively. In fact, $\mathrm{NC}$ has not been reported in linear systems below microwave frequencies with good reason: the parameter $\tau$ should be interpreted as the carrier relaxation time $\ll 10^{-9} \mathrm{~s}$, and lead to an $\omega C$ term negligibly small against the in-phase $1 / R_{\mathrm{ac}}{ }^{31,32}$ Some reports have proposed mechanisms, with varying degrees of rigor, behind the $\mathrm{NC}$ for the particular case in question. The explanations of these observances, naturally, are as varied as the materials in which they are found. Such divergent interpretations seem to be in strong contrast with the surprisingly similar features observed.

Previously, we have reported plasmalike NC in an electrorheological (ER) fluid. ${ }^{20,21}$ Here, we exploit the abovementioned common features to demonstrate the basic requirements necessary for the manifestation of NC. We show that an off-phase signal, arising from dc bias and ac signal mixing across a dispersive nonlinear element, is responsible for NC. Our mechanism allows us to predict the NC value of the ER fluid by merely examining the nonlinearity of the in-phase conductivity. The validity of this model is further demonstrated on published data from a few unrelated mate- rials. Hence, seemingly unrelated instances of NC may be understood under a common framework. It is interesting to note that the $\mathrm{NC}$ under this model could naturally extend to the static limit if the nonlinearity persists into this regime. We will also argue that such NC should represent a negativedifferential dielectric constant if the associated nonlinearity and dispersion are bulk phenomena. It is hoped that this mechanism will provide a pathway in the search for materials with a negative static dielectric constant (NSDC), a property whose very existence has long been debated.

\section{RESULTS AND DISCUSSION}

\section{A. NC in the ER fluid}

The ER fluid is a colloid of $50 \mathrm{~nm}$ urea-coated $\mathrm{Ba}_{0.8} \mathrm{Rb}_{0.4} \mathrm{TiO}\left(C_{2} \mathrm{O}_{4}\right)_{2}$ nanoparticles suspended in silicone oil. ${ }^{33}$ Measurement cells for the fluid experiments were constructed of two parallel electrodes. The complex differential admittance $1 / R_{\mathrm{ac}}+\boldsymbol{i} \omega C_{\mathrm{ac}}=\mathbf{I}_{\mathrm{ac}} / \mathbf{V}_{\mathrm{ac}}$ was measured under a superimposed dc bias, $V_{\mathrm{dc}}$. The details of the ER fluid and the experiments have been reported before. ${ }^{20,21}$ A typical data subset over $10^{-5}-10^{3} \mathrm{~Hz}$ was measured in a period of 1-7 days after a stabilization period of several days. The uncertainty of the phase angle ranges from $0.01^{\circ}-0.05^{\circ}$ and is much smaller than the $\arctan \left(\omega C_{\mathrm{ac}} R_{\mathrm{ac}}\right)>0.5^{\circ}$ for the impedances reported here.

The $1 / R_{\mathrm{ac}}$ and $C_{\mathrm{ac}}$ of an ER cell are shown in Fig. 1. Two prompt features can be immediately noticed. Both $1 / R_{\mathrm{ac}}$ and $C_{\mathrm{ac}}$ are $V_{\mathrm{dc}}$ independent (linear ac response) and have a power-lawlike dispersion above $10^{3} \mathrm{~Hz}$ (lines in the figure). In other words, at high frequencies, both follow the so-called universal dielectric response (UDR), an empirical model for almost all highly disordered systems. ${ }^{34}$ At lower $\omega$, however, deviations almost simultaneously appear in the two channels. The conductance becomes $V_{\mathrm{dc}}$ dependent, i.e., with nonlinear $I-V$ characteristics. The capacitance first deviates from the 


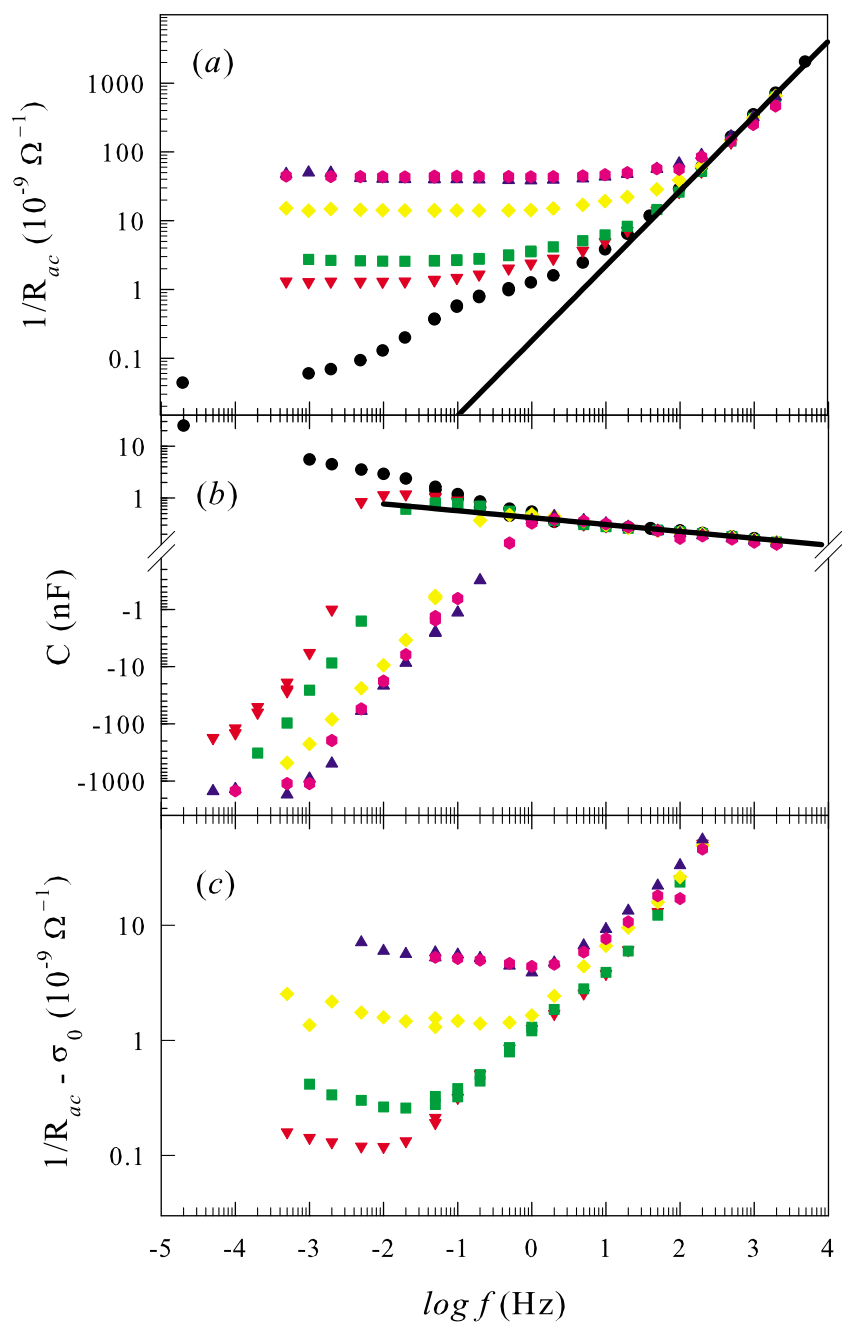

FIG. 1. (Color online) (a) The conductance of an ER cell under various dc biases. $0 \mathrm{~V}(\bullet), 50 \mathrm{~V}(\boldsymbol{\nabla}), 100 \mathrm{~V}(\boldsymbol{\nabla}), 200 \mathrm{~V}(\bullet), 350$ $\mathrm{V}(\boldsymbol{\Delta}), 500 \mathrm{~V}(\boldsymbol{\bullet})$. (b) The capacitances of an ER cell. (c) The conductance minimums in the low- $\omega$ region. $A_{0}=0.9^{*} 1 / R_{\max }\left(V_{\mathrm{dc}}\right)$ is a baseline subtracted for clarity.

power-law fit and becomes negative at lower $\omega$. A closer look further reveals that all $1 / R_{\mathrm{ac}}$ 's at a fixed $V_{\mathrm{dc}} \neq 0$ vary with $\omega$ nonmonotonically. To show this, an $\omega$-independent baseline $A_{0}$ was subtracted from $1 / R_{\mathrm{ac}}[$ Fig. $1(\mathrm{c})] .{ }^{20}$ It is clear by comparing Figs. $1(\mathrm{~b})$ and $1(\mathrm{c})$ that a $1 / R_{\mathrm{ac}}$ dip occurs around the same $\omega_{\mathrm{s}}$, where the negative $C(\omega)$ appears. This may not be an accidental coincidence. As previously demonstrated ${ }^{20}$ the analyticity of the complex ac response $1 / R_{\mathrm{ac}}+\boldsymbol{i} \omega C_{\mathrm{ac}}$ demands that a negative $C(\omega)$ and the associated $1 / R_{\mathrm{ac}}$ share a common plasmalike dispersion of $\propto 1 /\left[1+(\omega \tau)^{2}\right]$. Combined with the smooth increase in the conductance with $\omega$ predicated by the UDR model at higher frequencies, this component will naturally lead to a dip at $\omega_{\mathrm{s}}$. What has not been expected, however, is that a sudden enhancement of the $1 / R_{\mathrm{ac}}$ splitting with $V_{\mathrm{dc}}$, i.e., the nonlinearity, appears right below $\omega_{\mathrm{s}}$. This observation motivated us to look into the correlation between negative $C(\omega)$ and the nonlinearity.

\section{B. A general NC mechanism}

Such a correlation, actually, is surprisingly general. It is well known that a nonlinear sample, e.g., a diode, should behave linearly in the small-signal limit if a single sine-wave signal $V_{1} \sin \left(\omega_{1} t\right)$ is applied. All higher harmonics disappear with $V_{1} \rightarrow 0$, and Fourier transformations are perfectly legal if a differential admittance is used. The situation, however, may be different if there is another superimposed input of $V_{2} \sin \left(\omega_{2} t+\theta\right)$, such as in the widely used electronic multiplying mixers. In particular, a dc bias, the special case of $V_{2}=V_{\mathrm{dc}}, \omega_{2}=0$, and $\theta=\pi / 2$, can change the situation. For a dispersionless conductor, the bias current $I_{\mathrm{dc}}=G \cdot V_{\mathrm{dc}}$ will be modulated by the superimposed ac excitation through the conductance $G \approx G_{0}+\partial G / \partial V \cdot d V=G_{0}+\partial G / \partial V \cdot V_{1} \sin \left(\omega_{1} t\right)$ in the small-signal limit, where the ac response is perfectly linear. The second term, the modulation conductance $G_{1}=V_{\mathrm{dc}} \partial G / \partial V$, modulates the "dc" current such that the total differential conductance is $1 / R_{\mathrm{ac}}=G_{0}+G_{1}$ instead of the zero-order conductance $G_{0}=I_{\mathrm{dc}} / V_{\mathrm{dc}}$ alone in linear conductors. These are exact relationships for dispersionless conductors but represent a good approximation if the dispersion is weak, e.g., in the case of the ER fluid, the $1 / R_{\text {ac }}$ deviates from its quasistatic limit less than $10 \%$ below $1 \mathrm{~Hz}$ [Fig. $1(\mathrm{a})]$. In the presence of strong frequency dependences, one may reasonably assume that the $1 / R_{\mathrm{ac}}$ can still be decomposed as the sum of a conductance $G_{1}$ associated with the modulated $I_{\mathrm{dc}}$ and a zero-order conductance $G_{0}$ associated with the differential carrier mobility at $\omega$ and $V_{\text {dc }}$, i.e., that of an equivalent linear system. The corresponding $G_{0}$ and $G_{1}$ values, however, may be different. To highlight the underlying physics, we will first present the model with an explicit correlation between the negative capacitance and the nonlinearity $G_{1}=V_{\mathrm{dc}} \partial G / \partial V$. A modified, more practical, $G_{1}$ will then be proposed for analyzing actual experimental data, including situations in which noticeable dispersions exist.

It is interesting to note that any plasmalike dispersion in the conductance channel, including the modulation conductance, results in an additional off-phase plasma term in the ac current. Many known modulated currents, in particular, are retarded against the applied voltage, e.g., through the minority-carrier migration across the depletion layer or delay due to poisoning (contamination) of electrodes. Such retarded current enhancements, however, are exactly the same ac response of an inductor (or a capacitor with $C<0$ ) but the associated time-scale $\tau$ is no longer the carrier relaxation time. Mathematically, this is equivalent to adding a complex phase factor, i.e., modifying the real $V_{\mathrm{dc}} \frac{\partial G}{\partial V}$ into a complex differential admittance $\mathbf{A}_{1}=\left|\mathbf{A}_{1}\right| e^{-i \theta(\omega)}$ with its real part $\operatorname{Re}\left[\mathbf{A}_{1}\right]=\left.V_{\mathrm{dc}} \frac{\partial G}{\partial V}\right|_{\omega}$. The net capacitance $C$, which is the sum of a geometric capacitance $C_{0}$ and a modulation capacitance $C_{1}=-\left.V_{\mathrm{dc}} \frac{\partial G}{\partial V}\right|_{\omega} \frac{\tan (\theta)}{\omega}$, can be negative if $\left.V_{\mathrm{dc}} \frac{\partial G}{\partial V}\right|_{\omega} \gg C_{0} \omega / \tan (\theta)>0$. In other words, the nonlinearity and the dc bias combine to provide an additional term to the ac current, which quite often lags behind the voltage. This retarded current is manifested as a negative contribution to the capacitance. A negative capacitance appears when this contribution becomes dominant. The requirements of this mechanism are very general. However, a host of microscopic phenomena can produce the above-mentioned effects, thus, 
explaining the wide array of materials that exhibit NC.

To be quantitative, one still needs to reformulate it such that $C_{1}$ can be directly deduced from the measured modulated conductance $G_{1}=\operatorname{Re}\left[\mathbf{A}_{1}\right]=\left.V_{\mathrm{dc}} \frac{\partial G}{\partial V}\right|_{\omega}$. We write the frequency dependence explicitly as, $G_{1}=\left.V_{\mathrm{dc}} \frac{\partial G}{\partial V}\right|_{\omega=0} F^{\prime}(\omega)$, where $F^{\prime}(\omega)$ is the real part of a complex function describing the dispersion of the nonlinearity. Fortunately, the complex response $\mathbf{A}_{1}$ is an analytical (holomorphic) function of $\omega$, i.e., fully differentiable against $\omega,{ }^{35}$ in most practical cases. Thus, the corresponding contribution to the capacitance, $C_{1}=\left.V_{\mathrm{dc}} \frac{\partial G}{\partial V}\right|_{\omega=0} F^{\prime \prime}(\omega) / \omega$, can be deduced from $G_{1}$ by solving the Cauchy-Riemann equations for $F^{\prime \prime}(\omega) .{ }^{35}$ This is the most general representation of the mechanism. The relationship between $G_{1}$ and the NC can be seen more clearly for the case of a plasmalike relaxation. ${ }^{20}$ In such a case, the modulation conductance is $G_{1}=\left.V_{\mathrm{dc}} \frac{\partial G}{\partial V}\right|_{\omega=0} \frac{1}{1+(\omega \tau)^{2}}$. The contribution to the capacitance associated with this conductance is $C_{1}=-\left.V_{\mathrm{dc}} \frac{\partial G}{\partial V}\right|_{\omega=0} \frac{\tau}{1+(\omega \tau)^{2}}$. With $\left.\frac{\partial G}{\partial V}\right|_{\omega=0}>0$, such a term will yield a negative contribution to the capacitance, and as is often the case, become dominant at low frequencies. It is clear that, in order to calculate this effect on the capacitance, one only needs knowledge of the material's nonlinearity.

Dispersion may shift the $G_{1}$ value from its quasistatic limit of $\left.V_{\mathrm{dc}} \frac{\partial G}{\partial V}\right|_{\omega \rightarrow 0}$. As stated above, $\mathrm{NC}$ is often found in nonlinear materials, but, to our knowledge, there have been no reports of low-frequency $\mathrm{NC}$ in linear systems. ${ }^{31,32}$ It stands to reason that any plasmalike dispersion would reside in $G_{1}$ rather than $G_{0}$, the equivalent linear system. Furthermore, at low frequencies, most common dispersions, such as UDR or polarization relaxations, are relatively minor. This is clear, for example, in the ER fluid below $1 \mathrm{~Hz}$. Without inducing much error, therefore, we can conveniently set $G_{0}=I_{\mathrm{dc}} / V_{\mathrm{dc}}$. In this way, $G_{1}=1 / R_{\mathrm{ac}}-I_{\mathrm{dc}} / V_{\mathrm{dc}}$. The associated $\mathrm{NC}$, therefore, can be straightforwardly deduced from experimentally measurable parameters, $1 / R_{\mathrm{ac}}$ and $I_{\mathrm{dc}}$. This algebra, it should be pointed out, has an additional advantage of a lower noise floor since numerical calculation of the derivative $G_{1}=V_{\mathrm{dc}} \frac{\partial G}{\partial V}$ involves many data subsets at different dc biases. The low- $\omega$ conductivity of the ER fluid is highly sensitive to changes in room temperature and, particularly, rearrangement of nanoparticles while under bias. Lowfrequency measurements at various biases take several weeks to complete. During this period, such effects contribute to conductance drifts which interfere with direct calculation of $\frac{\partial G}{\partial V}$. This algebra, therefore, significantly suppresses the effects of the drifts at low frequencies by involving only the concurrently measured $R_{\mathrm{ac}}$ and $I_{\mathrm{dc}}$. In the ER fluid, far above $\omega_{\mathrm{s}}$, the strong UDR-like dispersion makes the situation complicated. $G_{0}$ can no longer to be assumed to be dispersionless over such frequency range. Fortunately, the nonlinearity is rather weak there. A $G_{0} \approx \int_{0}^{V_{\mathrm{dc}}} d V^{\prime} / R_{\mathrm{ac}}\left(V^{\prime}, \omega\right) / V_{\mathrm{dc}}$, which is exact in linear systems, is adopted. The consequences of such an approximation should be rather small since the negative capacitance is only marginally dependent on the $G_{1}$ values at high frequencies.

The nonlinearity, $\left.V_{\mathrm{dc}} \frac{\partial G}{\partial V}\right|_{\omega}$, of the ER fluid at $50 \mathrm{~V}$ [Fig. 2(a)] was deduced by the method described above for frequencies below $0.1 \mathrm{~Hz}$ (open circles) and by numerical integration above $10 \mathrm{~Hz}$ (open triangles). The nonlinearity was

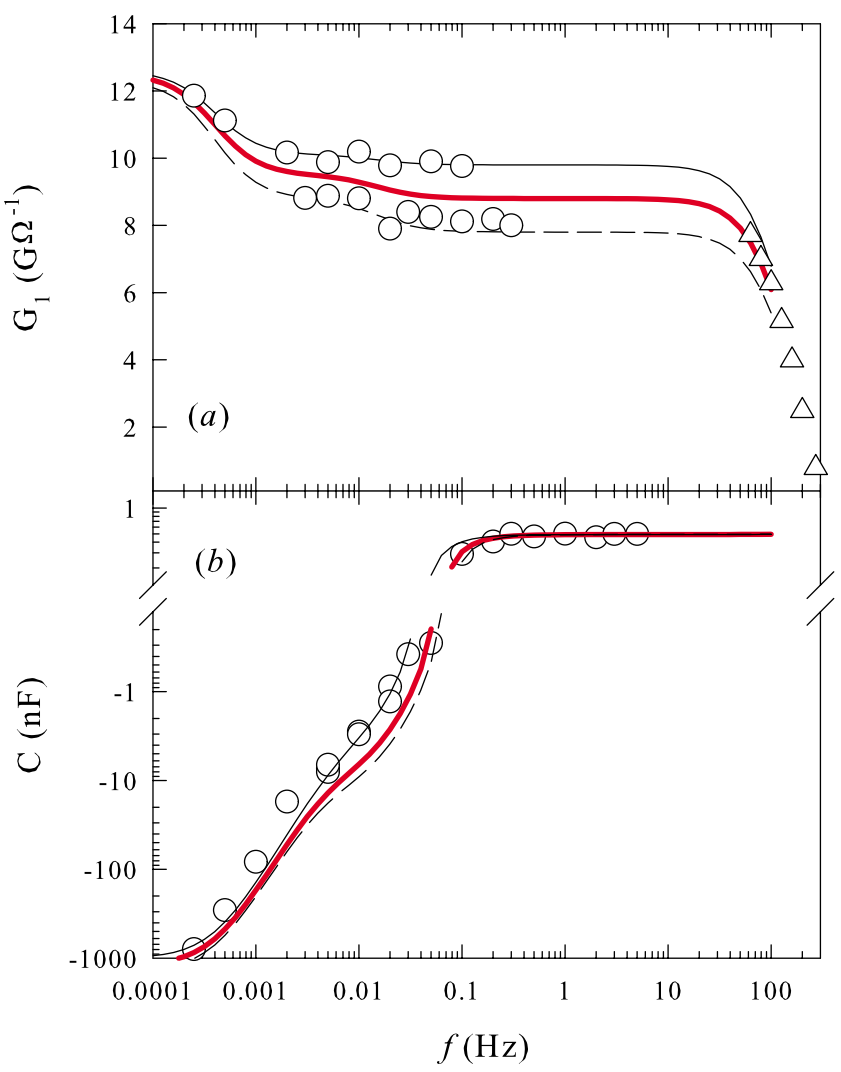

FIG. 2. (Color online) (a) The deduced modulation conductance (nonlinearity) of an ER fluid at $V_{\mathrm{dc}}=50 \mathrm{~V}$. The symbols are the measured data and the lines are the fits with three superimposed plasmalike relaxations. The lines represent the best fit and the upper and lower boundaries. The data scattering is much larger than the repeatability of the measured $1 / R_{\mathrm{ac}}$ due to the cancellation of $\frac{1}{R_{\mathrm{ac}}(\omega)}$ and $\frac{I_{\mathrm{dc}}}{V_{\mathrm{dc}}}$. This $\pm 15 \%$ uncertainty, fortunately, still allows a reasonable prediction due to the broad dynamic range of the $C$. (b) The capacitance under the same conditions. The symbols are the data. The lines are the predictions from the model using the nonlinearity deduced from Fig. 2(a).

then fit with three relaxation-time constants, i.e., as $\sum_{i=1 \ldots 3} \frac{a_{i}}{\left[1+\left(f / f_{i}\right)^{2}\right]}$ with $a_{i}$ and $f_{i}$ as fitting parameters. Three fits were used: one for the averaged data (central line), two as the upper and lower boundaries of the data (outer lines). The predicted $C_{\mathrm{ac}}(\omega)=\sum_{i=1 \ldots 3} \frac{a_{i}}{\left[1+\left(f / f_{i}\right)^{2}\right] f_{i}}+5 \times 10^{-10}$ is represented in Fig. 2(b), where the constant $5 \times 10^{-10}$ represent the high- $\omega$ geometric capacitance. The agreement is reasonably good, especially considering the dynamic range of $C_{\mathrm{ac}}$.

It should be emphasized that this is not a mathematical fit for the measured capacitances. The fit is only for the "passive" modulation conductance. Analycity constrains the $C(\omega)$ only with $1 / R_{\mathrm{ac}}$. Aside from the mechanism under discussion, there should be no other mathematical correlation between the nonlinearity and the capacitance. This is clear from the data above $100 \mathrm{~Hz}$. The negligibly small nonlinearity leads only to the UDR-like capacitance enhancement despite the strong $1 / R_{\mathrm{ac}}$ dispersion there. This good prediction, therefore, verifies the mechanism, in our opinion.

It is interesting to note (as discussed above) that almost all reported "negative capacitance" involve: dc bias, superlinear 


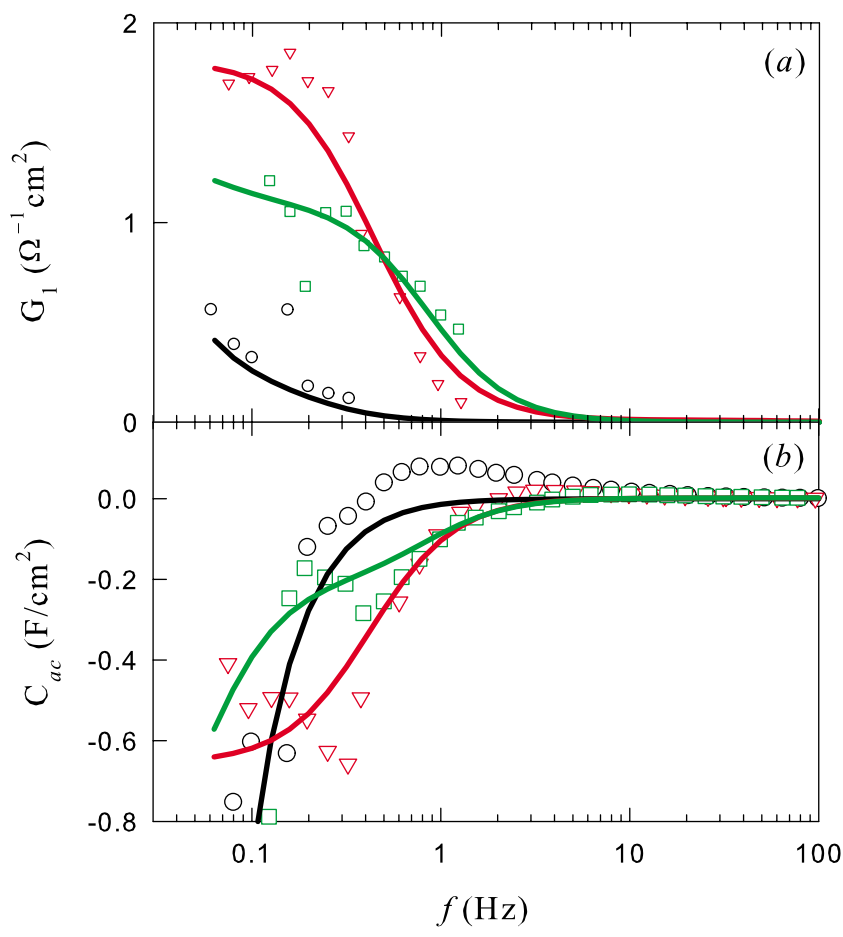

FIG. 3. (Color online) (a) The deduced modulation conductance (nonlinearity) of a $\mathrm{H}_{2} / 2 \% \mathrm{CO}+\mathrm{H}_{2}$ PEM fuel cell (Ref. 23). The symbols are the measured data. $\bigcirc$ : at $0.372 \mathrm{~V}, \nabla$ : at $0.528 \mathrm{~V}$, and $\square$ : at $0.62 \mathrm{~V}$. The respective lines are the fits with two superimposed plasmalike relaxations. (b) The capacitances under the same conditions. The symbols are the data. The lines are the predictions from the model.

$I-V$ characteristics and significant dispersion. The proposed "passive" mechanism, therefore, should contribute to the $\mathrm{NC}$, in principle. Whether it is the dominant one, however, needs detailed verification. Unfortunately, the information available in the literature is limited, thus prohibiting a quantitative reanalysis in most cases. Such reanalysis, nevertheless, has been done in a few instances. The data for a $\left(\mathrm{H}_{2} / \mathrm{H}_{2}+2 \% \mathrm{CO}\right)$ proton exchange membrane fuel cell ${ }^{23}$ are shown in Fig. 3. The differential conductance and capacitance (various symbols) were taken from the Fig. 6 of Ref. 23 , and $G_{0}$ at $0.125 \mathrm{~Hz}$ from integrating the data of the Fig. 9. Two excitations were used to fit the deduced nonlinearity [various lines in Fig. 3(a)]. The predicated NC agrees with the data reasonably well considering the limited data reported. The physical/chemical processes of fuel cells and ER fluids are very different. However, both exhibit NC which can be described by the model. Similar agreement was found in solar-cell data reported in Ref. 22. It appears, therefore, that the mechanism is rather a general one.

\section{Relationship between NC and the dielectric constant}

One of the key issues concerning the negative capacitance is whether it can be associated with a negative dielectric constant. Almost all previous publications carefully use the term "negative capacitance" and avoid connecting it with a dielectric constant. This is understandable when referring to devices such as diodes, where the NC originates at an inter- face within the device or an electrode, as is the case in the previously mentioned fuel cell. ${ }^{23}$ However, when NC is due to bulk processes, the use of a dielectric constant becomes appropriate. In the simplest case of a homogeneous medium, the dielectric constant $\varepsilon^{\prime}$ is defined in the Maxwell equations as the ratio of the "external" charge-density $\rho_{\text {ext }}$ and the total charge-density $\rho_{\text {tot }}$ in $\boldsymbol{\nabla} \cdot(\boldsymbol{\varepsilon} \mathbf{E})=4 \pi \rho_{\text {ext }}$ and $\boldsymbol{\nabla} \cdot \mathbf{E}=4 \pi \rho_{\text {tot }}$. To apply these to a highly conductive medium, however, may not be straightforward. A similar question, fortunately, has been well addressed for classical free-carrier plasmas. ${ }^{36}$ It is claimed in Ref. 36 that the $\varepsilon_{1}^{\prime}$ defined in the admittance, $\boldsymbol{\nabla} \cdot \mathbf{j}=\left(\sigma+i \omega \varepsilon_{1}^{\prime}\right) \boldsymbol{\nabla} \cdot \mathbf{E}$, of a conducting medium should be exactly the same as the dielectric constant $\varepsilon^{\prime}$ in the Maxwell equation $\left(\sigma+i \omega \varepsilon^{\prime}\right) \boldsymbol{\nabla} \cdot \mathbf{E}=4 \pi \rho_{\mathrm{ext}}$ in the $\omega$ domain. The authors of Ref. 36 further demonstrated that this equality should hold regardless the possible dispersions and medium anisotropy as long as the Fourier transformation is permitted. Strict linearity and Fourier transformation, however, are usually not a problem in the differential sense even if the overall $I-V$ characteristic is nonlinear. In such a situation the differential capacitance would be associated with a differential dielectric constant. To determine whether the NC in a particular case represents a negative-differential dielectric constant, therefore, one merely needs to exclude interface phenomena. According to the above model, the dominant $\mathrm{NC}$ mechanism should be the same one controlling $G_{1}$, e.g., which bears most of the voltage drop. All known electrode potentials, however, are $\ll 5 \mathrm{~V}$, so nonlinear resistance across the electrode interfaces is unlikely to significantly influence the ER cells. Indeed, the same NC data are observed using electrodes of various metal electrodes such as, $\mathrm{Cu}, \mathrm{Au}, \mathrm{Ni}, \mathrm{Pt}$ as well as indium tin oxide. However, in order to be conclusive, a direct comparison was made. The standard method of detecting the presence of dominant interfacial processes is to measure the impedance of samples possessing different thicknesses. ${ }^{37}$ If the impedance is dominated by bulk processes, it should scale with sample thickness. ${ }^{38}$ However, an impedance dominated by interfacial effects should be unaffected by a change in thickness. Thus, two, almost identical cells, A and B, were created with the sole difference being the distance, $d_{A}$ and $d_{B}$, between the electrodes. Highfrequency capacitance measurements indicate that the gap of cell $\mathrm{B}$ is approximately 1.8 times of that of cell $\mathrm{A}$, where $d_{A} \approx 0.1 \mathrm{~mm}$. It should be noted that the NC depends on the $\mathrm{dc}$ bias so strongly, e.g., with a rate of 3-6\%/V for both its low- $\omega$ value and its onset frequency around $V_{\mathrm{dc}}=50 \mathrm{~V}$ for cell A (Fig. 1), that it offers a good probe. If the $\mathrm{NC}$ is controlled by electrode reactions, then one would expect that the ratio $\frac{C_{B}}{C_{A}}$ equals to one if both cells are under the same bias, where $C_{A}$ and $C_{B}$ are the low- $\omega$ limits of $\mathrm{NC}$ for cells A and $\mathrm{B}$, respectively. However, if the nonlinearity originates in the bulk, the ratio $\frac{C_{B} \cdot d_{B}}{C_{A} \cdot d_{A}}$ will be equal to one only if the electric fields are the same in the two cells. In mixed cases, not only is such a match impossible under the above conditions but the $\omega$ dependency will also be distorted. For example, the ratio $\frac{C_{B}}{C_{A}}$ will equal to $\left(\frac{d_{A}}{d_{B}}\right)^{2}$, if the NC is controlled by the electrode reactions but the largest voltage drop occurs across the ER bulk. Cell B was, therefore, measured under various biases $U$ and compared with the cell A data at $50 \mathrm{~V}$. 


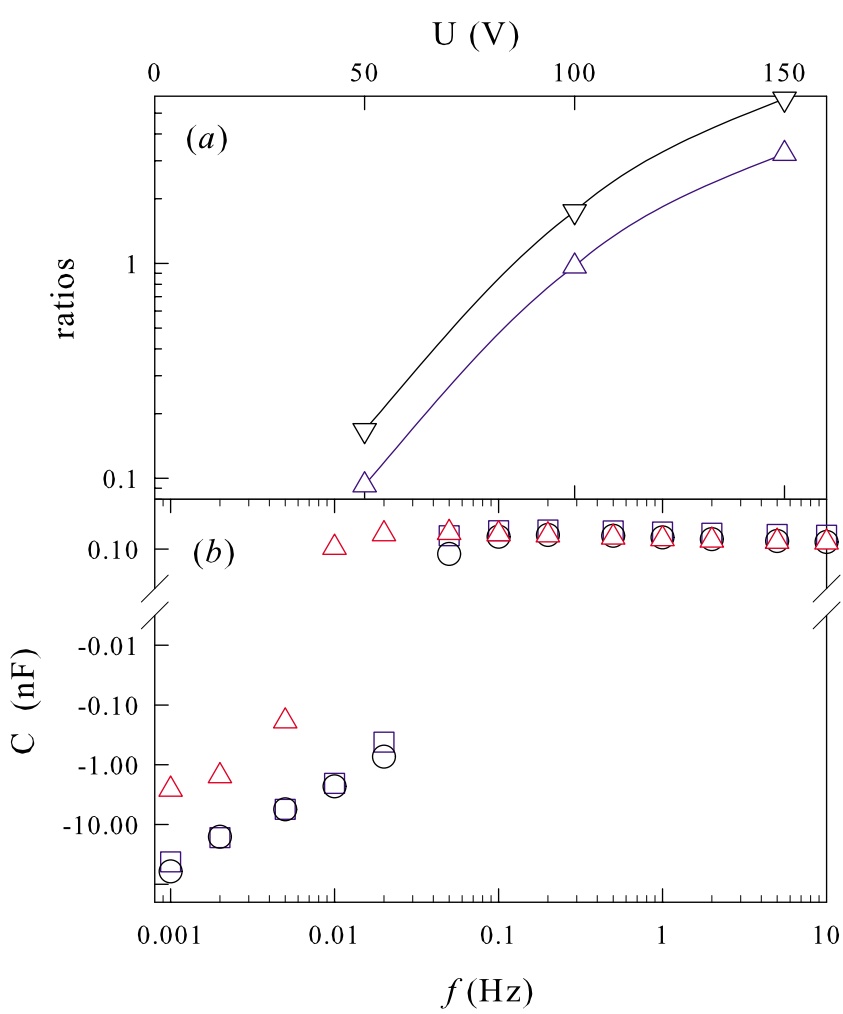

FIG. 4. (Color online) (a) The ratios $\frac{C_{B} \cdot d_{B}}{C_{A} \cdot d_{A}}(\nabla)$ and $\frac{C_{B}}{C_{A}}(\triangle)$ at 2 $\mathrm{mHz}$. The dc bias voltages are $50 \mathrm{~V}$ and $U$ for the cells $\mathrm{A}$ and $\mathrm{B}$, respectively. (b) The $C$ of cell A at $50 \mathrm{~V}(\bigcirc)$, cell B at $U=50 \mathrm{~V}(\triangle)$ and $100 \mathrm{~V}(\square)$.

The $\frac{C_{B}}{C_{A}}$ is only 0.09 with $U=50 \mathrm{~V}$ [Fig. 4(a)], i.e., ten times lower than that expected for the electrode effects. The interpolated $\frac{C_{B} \cdot d_{B}}{C_{A} \cdot d_{A}}=1.2$ at $U=90 \mathrm{~V}[$ Fig. 4(a)], on the other hand, is in rough agreement with that expected from the bulk effects. The $C(\omega)$ of cell B at a slightly higher $U=100 \mathrm{~V}$, in addition, is almost the same as that of cell A. The data of the two cells at the same dc bias, however, are very different. The data, therefore, demonstrate that the NC observed here is a bulk phenomenon. Thus, the ER fluid is linear in the small signal limit and has an off-phase current that originates in the bulk of the fluid. Therefore, it is natural to associate the resulting capacitance, positive or negative, with a differential dielectric constant.

It is interesting to consider whether this bulk phenomenon can offer any insight about a NSDC. This has been a long debated and puzzling topic. A material possessing NSDC, for example, will allow for carriers of like charge to be mutually attracted. It has also been proposed that the transition temperature of the phonon-mediated superconductors can be much higher than $20-30 \mathrm{~K}$ only if NSDC is permitted. ${ }^{39}$ Whether NSDC is permitted and actually exists in a real material, however, is debatable. The situation on the theoretical side, fortunately, is much clearer now. The early doubt of whether the causality and lattice instability will fundamentally prohibit NSDC is largely settled. ${ }^{39,40}$ The experimental verification, however, is far from satisfactory.

With the concept of a NSDC in mind, we have extended our measurement range to very low frequencies in order to study the static limit of the fluid's dielectric constant, albeit in the differential sense. The issue that needs to be addressed is whether the dielectric constant observed down to $10^{-5} \mathrm{~Hz}$ can be extrapolated to the static limit, which is, in principle, unreachable experimentally within a finite measurement period. Under the above mechanism, however, the negative capacitance is the result of the nonlinearity in the conductance channel. While the exact microscopic mechanism responsible for this nonlinearity may change from one case to another, there is little reason to expect such nonlinearity can be drastically and totally suppressed over $0<\omega<10^{-4}$. If the $1 / R_{\mathrm{ac}}$ change in the ER cells were the result of the $E$-induced pressure between adjacent particles, for example, there is no clear reason why it cannot be kept at the static limit. This "passive" mechanism, therefore, offers a way to realize a negative static differential dielectric constant. The nonlinearity model and the ER-fluid data discussed above, hopefully, may open avenues into the investigations of NSDC and its possible applications.

\section{CONCLUSIONS}

In summary, a mechanism is proposed to describe the origin of the negative capacitance in the fluid. It states that a negative capacitance should appear while (a) a dc bias is superimposed; (b) the $I-V$ characteristic is superlinear; and (c) relaxations are associated with the nonlinearity. The NC results from an off phase component of the current which arises due to the combination of the dc bias and the nonlinearity. The applicability of the mechanism is quite general, and it is suggested to be valid for a wide range of $\mathrm{NCs}$ observed across a variety of materials. The abstract nature of $\mathrm{NC}$ can hinder investigations of these materials. However, in light of the mechanism, questions about the origin of $\mathrm{NC}$ in a particular material are now equivalent to asking why the material is dispersive and nonlinear. Dispersion and nonlinearity are much more tangible, as well as accessible, than NC itself. Thus, the mechanism provides a significant simplification to the original problem. Furthermore, dispersion and nonlinearity can be attained through many microscopic processes. The early puzzle of the many physically distinct materials having similar manifestations of NC is, therefore, explained by the mechanism. Under certain conditions, like those associated with the ER fluid, the low-frequency negative capacitances due to such a mechanism can be associated with a negative static differential dielectric constant.

\section{ACKNOWLEDGMENTS}

The work in Houston is supported in part by NSF under Grant No. DMR-9804325, the U. S. Air Force Office of Scientific Research, the T. L. L. Temple Foundation, the John J. and Rebecca Moores Endowment, and the State of Texas through the Texas Center for Superconductivity at the University of Houston; and at Lawrence Berkeley Laboratory by the Director, Office of Science, Office of Basic Energy Sciences, Division of Materials Sciences and Engineering of the U.S. Department of Energy under Contract No. DE-AC03$76 \mathrm{SF} 00098$. 
*jshulman@uh.edu

${ }^{1}$ N. A. Penin, Semiconductors 30, 340 (1996).

${ }^{2}$ A. G. U. Perera, W. Z. Shen, M. Ershov, H. C. Liu, M. Buchanan, and W. J. Schaff, Appl. Phys. Lett. 74, 3167 (1999).

${ }^{3}$ C. D. Wang, C. Y. Zhu, G. Y. Zhang, J. Shen, and L. Li, IEEE Trans. Electron Devices 50, 1145 (2003).

${ }^{4}$ C. Y. Zhu, C. D. Wang, L. F. Feng, G. Y. Zhang, L. S. Yu, and J. Shen, Solid-State Electron. 50, 821 (2006).

${ }^{5}$ S. T. Mahmoud, H. Ghamlouche, N. Qamhieh, and S. Ahmed, J. Non-Cryst. Solids 354, 1976 (2008).

${ }^{6}$ M. Matsumura and Y. Hirose, Jpn. J. Appl. Phys. 39, L123 (2000).

${ }^{7}$ J. Werner, A. F. J. Levi, R. T. Tung, M. Anzlowar, and M. Pinto, Phys. Rev. Lett. 60, 53 (1988).

${ }^{8}$ S. H. Zaidi and A. K. Jonscher, Semicond. Sci. Technol. 2, 587 (1987).

${ }^{9}$ M. Ershov, H. C. Liu, L. Li, M. Buchanan, Z. R. Wasilewski, and V. Ryzhii, Appl. Phys. Lett. 70, 1828 (1997).

${ }^{10}$ H. H. P. Gommans, M. Kemerink, and R. A. J. Janssen, Phys. Rev. B 72, 235204 (2005).

${ }^{11}$ L. S. C. Pingree, B. J. Scott, M. T. Russell, T. J. Marks, and M. C. Hersam, Appl. Phys. Lett. 86, 073509 (2005).

${ }^{12}$ L. S. C. Pingree, M. T. Russell, T. J. Marks, and M. C. Hersam, J. Appl. Phys. 100, 044502 (2006).

${ }^{13}$ J. Bisquert, G. Garcia-Belmonte, A. Pitarch, and H. J. Bolink, Chem. Phys. Lett. 422, 184 (2006).

${ }^{14}$ F. A. Castro, P. R. Bueno, C. F. O. Graeff, F. Nüesch, L. Zuppiroli, L. F. Santos, and R. M. Faria, Appl. Phys. Lett. 87, 013505 (2005).

${ }^{15}$ V. Kytin, T. Dittrich, F. Koch, and E. Lebedev, Appl. Phys. Lett. 79, 108 (2001).

${ }^{16}$ L. Bakueva, G. Konstantatos, S. Musikhin, H. E. Ruda, and A. Shik, Appl. Phys. Lett. 85, 3567 (2004).

${ }^{17}$ C. Godet, J. P. Kleide, and A. S. Gudovskikh, J. Non-Cryst. Solids 354, 2637 (2008).

${ }^{18}$ G. B. Parravicini, A. Stella, M. C. Ungureanu, and R. Kofman, Appl. Phys. Lett. 85, 302 (2004).

${ }^{19}$ W. Huang, J. Peng, L. Wang, J. Wang, and Y. Cao, Appl. Phys. Lett. 92, 013308 (2008).

${ }^{20}$ J. Shulman, S. Tsui, F. Chen, Y. Y. Xue, and C. W. Chu, Appl.
Phys. Lett. 90, 032902 (2007).

${ }^{21}$ F. Chen, J. Shulman, S. Tsui, Y. Y. Xue, W. Wen, P. Sheng, and C. W. Chu, Philos. Mag. 86, 2393 (2006).

${ }^{22}$ I. Mora-Sero, Juan Bisquert, Francisco Fabregat-Santiago, Germà Garcia-Belmonte, Guillaume Zoppi, Ken Durose, Yuri Proskuryakov, Ilona Oja, Abdelhak Belaidi, Thomas Dittrich, Ramón Tena-Zaera, Abou Katty, Claude Lévy-Clément, Vincent Barrioz, and Stuart J. C. Irvine, Nano Lett. 6, 640 (2006).

${ }^{23}$ M. Ciureanu, H. Wang, and Z. Qi, J. Phys. Chem. B 103, 9645 (1999).

${ }^{24}$ F. Hajbolouri, B. Andreaus, G. G. Scherer, and A. Wokaun, Fuel Cells 4, 160 (2004).

${ }^{25}$ Z. Hens and W. P. Gomes, J. Phys. Chem. B 104, 7725 (2000).

${ }^{26}$ G. R. Olhoeft, Geophysics 50, 2492 (1985).

${ }^{27}$ R. P. Buck, Ann. Biomed. Eng. 20, 363 (1992).

${ }^{28}$ S. M. Ross, J. M. Ferrier, and J. Dainty, J. Membr. Biol. 85, 233 (1985).

${ }^{29}$ K. S. Cole, Membranes, Ions, and Impulses (University of California Press, Berkeley, 1968).

${ }^{30}$ E. R. Owede and A. K. Jonscher, J. Electrochem. Soc. 135, 1757 (1988).

${ }^{31}$ M. Scheffler, M. Dressel, M. Jourdan, and H. Adrian, Nature (London) 438, 1135 (2005).

${ }^{32}$ J. B. Pendry, A. J. Holden, W. J. Stewart, and I. Youngs, Phys. Rev. Lett. 76, 4773 (1996).

${ }^{33}$ W. Wen, X. Huang, S. Yang, K. Lu, and P. Sheng, Nature Mater. 2, 727 (2003).

${ }^{34}$ J. C. Dyre and T. B. Schroder, Rev. Mod. Phys. 72, 873 (2000).

${ }^{35}$ M. Boas, Mathematical Methods in the Physical Sciences, 2nd ed. (John Wiley \& Sons, New York, 1983), Chap. 14.

${ }^{36}$ M. G. Calkin and P. J. Nicholson, Rev. Mod. Phys. 39, 361 (1967).

${ }^{37}$ A. K. Jonsher, Dielectric Relaxation in Solids (Chelsea Dielectrics, London, 1983), p. 234.

${ }^{38}$ In the case of a nonlinear material the electric field must also be equal in samples of different thickness since the resistance and capacitance are field dependent.

${ }^{39}$ V. L. Ginzburg, Rev. Mod. Phys. 76, 981 (2004).

${ }^{40}$ O. V. Dolgov, D. A. Kirzhnits, and E. G. Maksimov, Rev. Mod. Phys. 53, 81 (1981). 